\title{
Intramedullary Spine Disease
}

One of the most significant impacts of magnetic resonance (MR) has been its ability to exquisitely depict normal and pathologic anatomy of the spine. Direct acquisitions acquired in multiple planes coupled with the ability to study the spine with different $T_{1^{-}}$ and $T_{2}$-weighted images have enabled critical assessment of the spinal cord and its surroundings not previously available to the medical imaging specialist. The development of contrast media has further extended the capability of MR imaging of the spinal cord by improving its sensitivity and has allowed the use of the method in certain additional types of pathology. The first protocol (see Basic Protocol) deals with intramedullary disease, i.e., that involving the cord, the next one deals with extramedullary-intradural disease. Both can cause myelopathy.

\section{MYELOPATHY}

Our basic protocol consists of a sagittal $T_{1}$-weighted conventional spin echo, a sagittal $T_{2}$-weighted fast spin echo, and a transverse $T_{2}{ }^{*}$-weighted gradient echo. Most of the time we will also add $T_{1}$-weighted images in the sagittal and transverse planes before and after administration of gadolinium. It is important that at least one pair of pre- and post-contrast images be identical to facilitate detection of subtle enhancement. Thus, if fat saturation is used following administration of contrast, it should also be used at least in one plane prior to injection of contrast agent. At $1.5 \mathrm{~T}$, we use a dose of $0.1 \mathrm{mmol} / \mathrm{kg}$; on our low field open magnet, we use $0.2 \mathrm{mmol} / \mathrm{kg}$ to get comparable enhancement. The reason for this is that $T_{1}$ increases with field strength so that, for a given $T_{\mathrm{R}}$ of $500 \mathrm{msec}$, the low field acquisition is actually less $T_{1}$-weighted than a high field acquisition. Coupled to this is the need for a longer $T_{\mathrm{E}}$ to accommodate the longer echo sampling time required for a lower bandwidth acquisition. The resultant increased $T_{2}$-weighting competes with one's already marginal $T_{1}$-weighted contrast—hence the need for double dose at lower field.

When the sensory level is fairly specific, we will limit the examination to the cervical or thoracic spine; for most myelopathy workups; however, we do the complete spinal cord from foramen magnum to conus. This can usually be accomplished in one acquisition using phased array coils without loss of signal-to-noise. The cervical and thoracic portions are then magnified and filmed (or viewed) separately. While we always acquire transverse images through the cervical spine, we generally only acquire transverse images at the level of a suspected abnormality in the thoracic cord.

Table A9.1.1 lists the hardware necessary to perform the procedure. Subsequent tables list imaging parameters appropriate for high field-i.e., 1.0 to $1.5 \mathrm{~T}$. For lower fields, the echo times (i.e., $T_{\mathrm{E}}$ ) are generally increased to accommodate lower bandwidths and the number of acquisitions $\left(N_{\text {acq }}\right)$ is generally doubled.

This protocol takes about $30 \mathrm{~min}$ from start to finish.

Table A9.1.1 Equipment Parameters

\begin{tabular}{ll}
\hline Coil type & Circularly polarized (quadrature) neck coil and/or \\
& torso phased array coil \\
Cardiac gating & No \\
Peripheral gating & For safety only \\
Respiratory gating & No \\
Respirator & If required by patient \\
Oxygen & If required by patient \\
Motion cushions & Useful \\
Use of contrast agents & Yes \\
\hline
\end{tabular}

Contributed by William G. Bradley

Current Protocols in Magnetic Resonance Imaging (2001) A9.1.1-A9.1.12

Copyright $\odot 2001$ by John Wiley \& Sons, Inc.

BASIC

PROTOCOL
Intradural Spine

A9.1.1 


\section{Materials}

Normal saline $(0.9 \% \mathrm{NaCl} ; 500-\mathrm{ml}$ bag $)$

K-50 tubing

23- to 25-G butterfly needle

Intravenous MRI contrast agent (e.g., Magnevist, Omniscan, Prohance, or

OptiMark at a dose of $0.1 \mathrm{mmol} / \mathrm{kg}$ for high field or $0.2 \mathrm{mmol} / \mathrm{kg}$ for low field).

NOTE: Be sure that technicians and nurses always have immediate access to any emergency equipment that may be relevant to a given study, or that may be needed for a particular patient-i.e., crash carts and oxygen.

\section{Set up equipment and patient}

1. Interview (screen) the patient to ensure that he or she has no contraindications such as cardiac pacemakers or other implants containing ferromagnetic materials. Also be sure to find out if the patient has any health conditions that may require the presence of special emergency equipment during the scanning procedure, or necessitate any other precautions.

Generally standard screening forms are used for all patients scanned in a magnetic resonance system.

The presence of any ferromagnetic metals may be a health hazard to the patient when he or she is inside the magnet, and will also affect the imaging. If in doubt as to the exact composition of the items, it is best to exclude patients with any metal implants; see Shellock (1996) for discussion of what implants may be safely scanned using magnetic resonance.

Patients may be accompanied into the magnet room by a friend or family member, who can sit in the room during the scan and comfort the patient as needed. This companion must be screened as well to ensure the absence of loose metal objects on the body or clothing.

2. If the procedure is a research protocol, have the patient sign any necessary consent form.

3. Have the patient remove all jewelry and change into a gown to eliminate any metal that might be found in clothing.

4. Inform the patient about what will occur during the procedure, what he or she will experience while in the magnet and how to behave, including the following.

a. If earphones or headphones are used to protect the ears from the loud sounds produced by the gradients, the patient will be asked to wear these, but will be able to communicate with you at any time during the imaging.

b. The patient will be given a safety squeeze-bulb or similar equipment to request assistance at any time (demonstrate how this works).

c. For good results the patient should not talk, and should avoid or minimize swallowing or other movement, during each scan-i.e., as long as the banging sounds continue. Between scans, talking and swallowing are allowed in most cases, but other motion should be avoided when comparative positional studies are being performed; the patient will be informed when this is the case.

d. Nevertheless, the patient may call out at any time if he or she feels it necessary.

5. Help the patient mount onto the table. Either before or right after the patient lies down, set up any triggering devices or other monitoring equipment that is to be used.

6. Center the coil over the region of the spine where the key information is desired. Make sure that the body is constrained to prevent motion, especially if high resolution scans are to be run. 
7. If intravenous gadolinium or i.v. sedation will be given, start an i.v. line and attach it securely to the patient so that movement into or out of the magnet will not pull at the patient's arm. Hang a bag of saline and adjust drip to keep it open.

It is preferable to insert the line prior to imaging and to leave the patient in the magnet, with no intervening motion, between the scans run before contrast agent injection and those run after injection.

8. If needed, place a pillow or other support under the head and knees to make the patient more comfortable.

9. Use the centering light to position the patient (Table A9.1.2) at the threshold of the magnet and then put him or her into the center of the magnet.

Once this step has been performed, so long as the patient does not move on the table, the table itself can be moved and then replaced in the same position as before without jeopardizing the positioning of one scan relative to another.

10. If the patient is unable to hold still, provide an appropriate sedative; if the patient is in pain, provide an appropriate analgesic.

\section{Sequence 1: Rapid positioning pilot (Fig. A9.1.1)}

11. To verify patient position, run the pilot (or scout) scan to ensure correct location of the neck or back in three dimensions, using the imaging sequence given in Table A9.1.2 or similar parameters.

This sequence usually consists of three orthogonal planes to allow subsequent localization. The images are often also used later to determine where to place the saturation pulses and to set up coverage of the volume of interest.

\section{Sequence 2: Sagittal $T_{1}$-weighted conventional spin echo (Fig. A9.1.2)}

12. Set the imaging parameters as shown in Table A9.1.3.

13. Use the pilot image to locate the spine in three dimensions to ensure coverage of the region of interest (e.g., cervical, thoracic, lumbosacral spine).

14. Tell the patient (through the speaker system) that you are ready to begin the scan.

Table A9.1.2 Primary Clinical Imaging Parameters for Pilot Scan (Sequence 1)

\begin{tabular}{ll}
\hline $\begin{array}{l}\text { Patient position } \\
\text { Scan type }\end{array}$ & $\begin{array}{l}\text { Supine } \\
\text { Gradient Echo }\end{array}$ \\
Imaging plane (orientation) & Transverse, sagittal, coronal \\
Central slice or volume center & Cervical: on thyroid cartilage \\
& Thoracic: on sternal notch \\
Echo time $\left(T_{\mathrm{E}}\right)$ & As short as possible \\
Repeat time $\left(T_{\mathrm{R}}\right)$ & As short as possible \\
Flip angle $(\mathrm{FA})$ & $15^{\circ}$ \\
Fields of view $\left(\mathrm{FOV}, \mathrm{F}_{\mathrm{x}}, \mathrm{FOV}\right)$ & Cervical: $280 \mathrm{~mm}, 280 \mathrm{~mm}$ \\
& Thoracic: $350 \mathrm{~mm}, 350 \mathrm{~mm}$ \\
Resolution $(\Delta x, \Delta y)$ & Cervical $1.09 \mathrm{~mm}, 2.19 \mathrm{~mm}$ \\
& Thoracic: $1.37 \mathrm{~mm}, 2.73 \mathrm{~mm}$ \\
Number of data points collected $\left(N_{\mathrm{x}}, N_{\mathrm{y}}\right)$ & 256,128 \\
Display matrix $\left(D_{\mathrm{x}}, D_{\mathrm{y}}\right)$ & 256,256 \\
Slice thickness $(\Delta z)$ & 5 mm \\
Number of slices & 5 in each plane \\
Slice gap & 1 mm \\
Number of acquisitions $\left(N_{\text {acq }}\right)$ & 1 \\
Scan time & 30 sec (all 3 planes $)$ \\
\hline
\end{tabular}


A

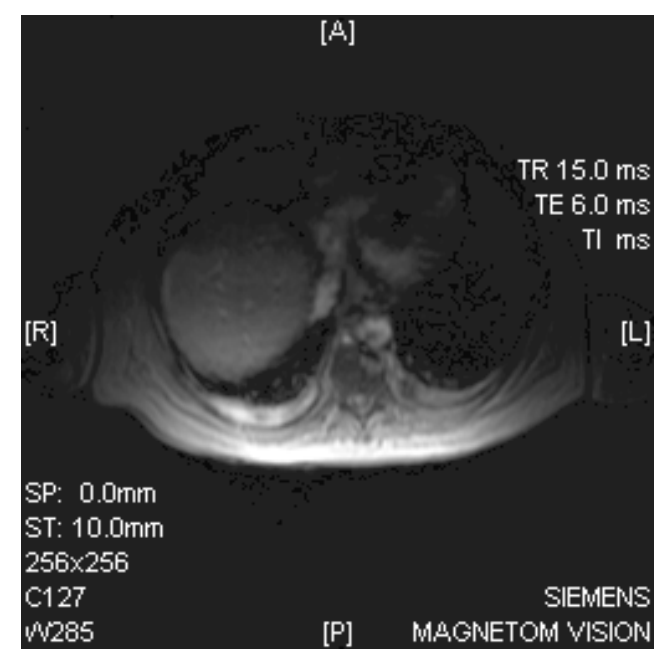

C

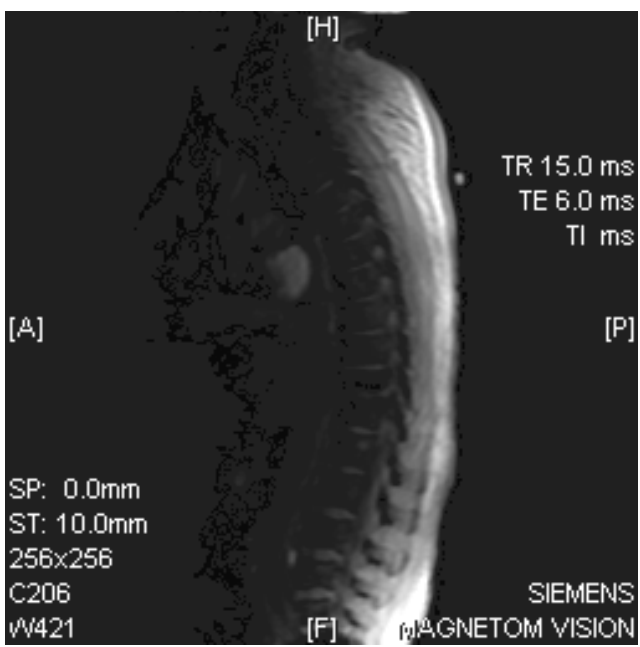

B

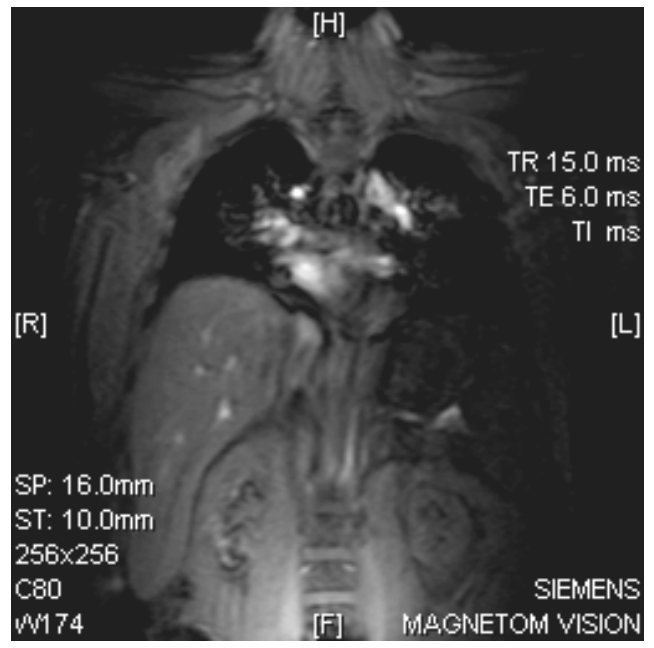

Intramedullary

Spine Disease

\section{Sequence 3: Sagittal $T_{2}$-weighted fast spin echo (FSE) (Fig. A9.1.3)}

15. Review the pilot scans and ensure the saturation pulses are correctly placed anterior to or above the slab of interest.

16. Run sequence 3 according to Table A9.1.4.

\section{Sequence 4: Transverse $T_{2}{ }^{*}$-weighted gradient echo (Fig. A9.1.4)}

17. Using the midline sagittal $T_{1}$-weighted image acquired in sequence 2 as a localizer:

a. Cervical spine: acquired stacked images from $\mathrm{C} 1$ through $\mathrm{T} 1$.

b. Thoracic spine: acquire single or stacked images through the levels of interest.

c. Supplement with additional slices according to visible disease present or to clinical query.

18. Run sequence 4 according to Table A9.1.5. 


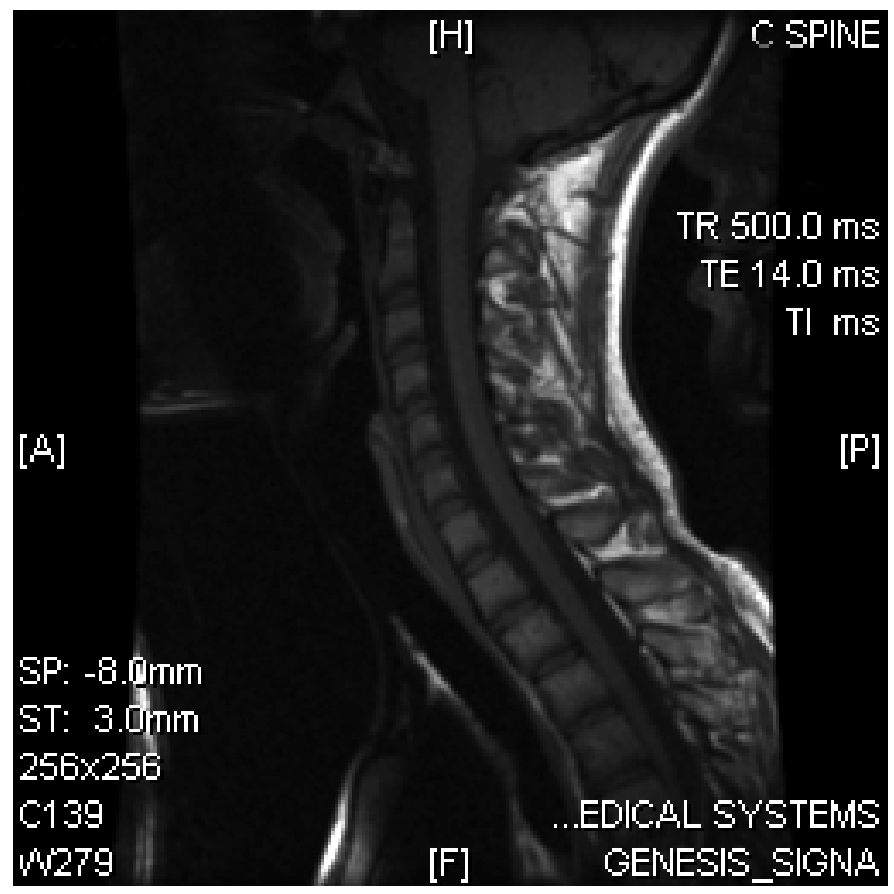

Figure A9.1.2 Sagittal $T_{1}$-weighted spin echo image.

Table A9.1.3 Primary Clinical Imaging Parameters for $T_{1}$-Weighted Spin Echo (Sequence 2)

Patient position

Scan type

Imaging plane (orientation)

Central slice or volume center

Echo time $\left(T_{\mathrm{E}}\right)$

Repeat time $\left(T_{\mathrm{R}}\right)$

Flip angle (FA)

Fields of view $\left(\mathrm{FOV}_{\mathrm{x}}, \mathrm{FOV}_{\mathrm{y}}\right)$

Resolution $(\Delta x, \Delta y)$

Number of data points collected $\left(N_{\mathrm{x}}, N_{\mathrm{y}}\right)$

Display matrix $\left(D_{\mathrm{x}}, D_{\mathrm{y}}\right)$

Slice thickness $(\Delta z)$

Number of slices

Slice gap

Number of acquisitions $\left(N_{\text {acq }}\right)$

Flow compensation

Saturation pulses

Scan time
Supine

Conventional spin echo

Sagittal

Slice centered on:

Cervical: the third cervical vertebra

Thoracic: the 6th thoracic vertebra $14 \mathrm{msec}$

$500 \mathrm{msec}$

$90^{\circ}$

Cervical: $260 \mathrm{~mm}, 260 \mathrm{~mm}$

Thoracic: $320 \mathrm{~mm}, 320 \mathrm{~mm}$ (may use rectangular field of view [e.g., half or three-quarter field] if available, or tailor to region of interest)

Cervical: $1.02 \mathrm{~mm}, 1.02 \mathrm{~mm}$

Thoracic: $1.25 \mathrm{~mm}, 1.25 \mathrm{~mm}$

256, 256

256,256

$3 \mathrm{~mm}$

12

$1 \mathrm{~mm}$

3

Yes (if available)

Superior, inferior, anterior $3 \mathrm{~min}, 20 \mathrm{sec}$ 


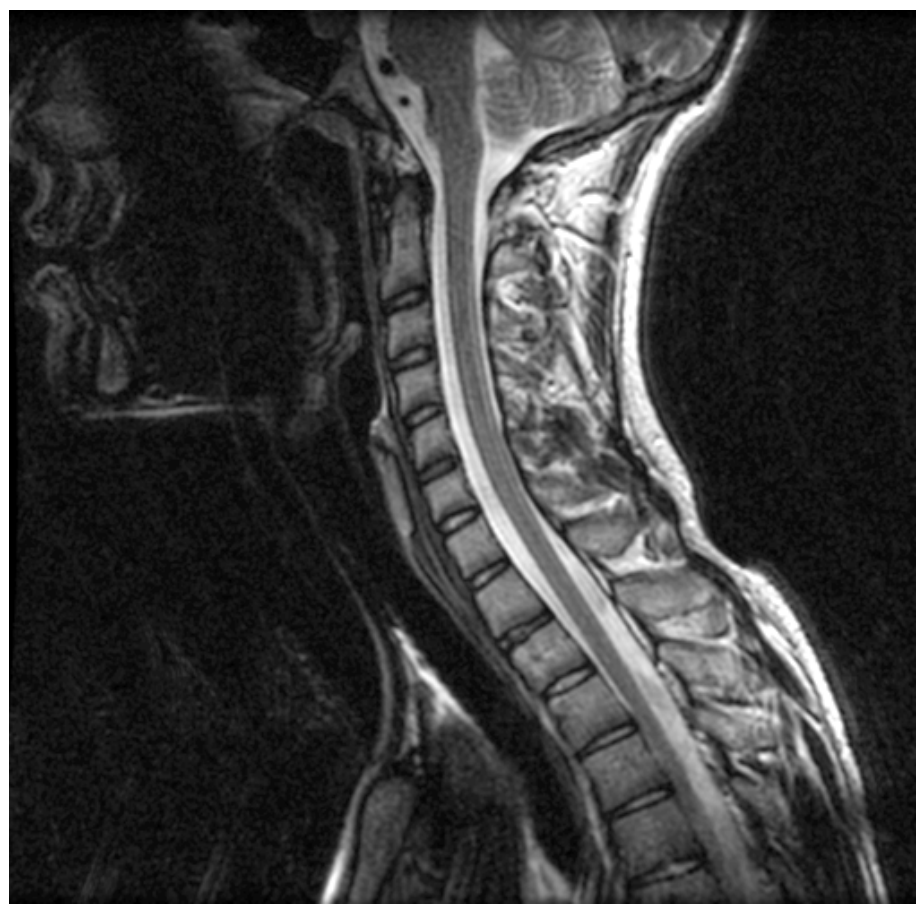

Figure A9.1.3 Sagittal $T_{2}$-weighted fast spin echo image.

Table A9.1.4 Primary Clinical Imaging Parameters for $T_{2}$-Weighted FSE (Sequence 3)

Patient position

Scan type

Imaging plane (orientation)

Central slice or volume center

Echo time $\left(T_{\mathrm{E}}\right)$

Echo train length (ETL)

Repeat time $\left(T_{\mathrm{R}}\right)$

Flip angle (FA)

Fields of view $\left(\mathrm{FOV}_{\mathrm{x}}, \mathrm{FOV}_{\mathrm{y}}\right)$

Resolution $(\Delta x, \Delta y)$

Number of data points collected $\left(N_{\mathrm{x}}, N_{\mathrm{y}}\right)$

Display matrix $\left(D_{\mathrm{x}}, D_{\mathrm{y}}\right)$

Slice thickness $(\Delta z)$

Number of slices

Slice gap

Number of acquisitions $\left(N_{\mathrm{acq}}\right)$

Flow compensation

Saturation pulses

Scan time
Supine

Fast spin echo

Sagittal

Centered on area of interest (as in sequence 2, Table A9.1.3)

$102 \mathrm{msec}$

8

$4000 \mathrm{msec}$

$90^{\circ}$

As in sequence 2, Table A9.1.3

As in sequence 2, Table A9.1.3

256, 256

256,256

$3 \mathrm{~mm}$

12

$1 \mathrm{~mm}$

3

Yes (if available)

Superior, inferior, anterior

$3 \mathrm{~min}, 12 \mathrm{sec}$ 


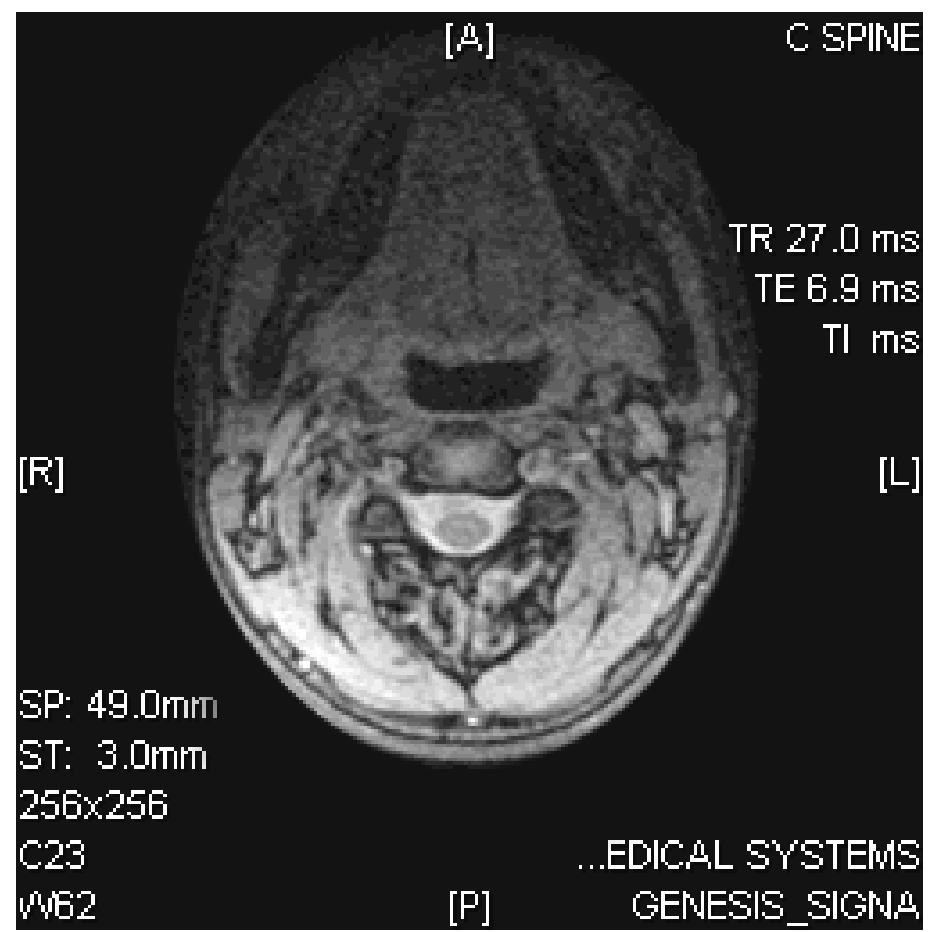

Figure A9.1.4 Transverse $T_{2}{ }^{*}$-weighted gradient echo image.

Table A9.1.5 Primary Clinical Imaging Parameters for $T_{2}{ }^{*}$-Weighted Gradient Echo (Sequence 4)

\begin{tabular}{ll}
\hline Patient position & Supine \\
Scan type & 3-D gradient echo \\
Imaging plane (orientation) & Transverse \\
Central slice or volume center & Volume centered on the area of \\
& interest (as in sequence 2, Table \\
Echo time $\left(T_{\mathrm{E}}\right)$ & A9.1.3) \\
Repeat time $\left(T_{\mathrm{R}}\right)$ & $16 \mathrm{msec}$ \\
Flip angle (FA) & $35 \mathrm{msec}$ \\
Fields of view $\left(\mathrm{FOV}, \mathrm{FOV}_{\mathrm{y}}\right)$ & $5^{\circ}$ \\
Resolution $(\Delta x, \Delta y)$ & $220 \mathrm{~mm}, 220 \mathrm{~mm}$ \\
Number of data points collected $\left(N_{\mathrm{x}}, N_{\mathrm{y}}\right)$ & $0.86 \mathrm{~mm}, 0.86 \mathrm{~mm}$ \\
Display matrix $\left(D_{\mathrm{x}}, D_{\mathrm{y}}\right)$ & 256,256 \\
Slice thickness $(\Delta z)$ & 256,256 \\
Number of slices & $2-3 \mathrm{~mm}{ }^{a}$ \\
Slice gap & 36 \\
Number of acquisitions $\left(N_{\mathrm{acq}}\right)$ & 0 \\
Flow compensation & 1 \\
Saturation pulses & Yes (if available) \\
Scan time & No \\
\hline af 3 min, 26 sec
\end{tabular}

${ }^{a}$ If $3 \mathrm{~mm}$, zero interpolate (ZIP) to $1.5 \mathrm{~mm}$. 
Sequence 5: Transverse $T_{1}$-weighted conventional spin echo (Fig. A9.1.5)

19. Using the midline $T_{1}$-weighted image acquired in sequence 2 as a localizer, position slices as in step 17 and run sequence 5 according to Table A9.1.6.

\section{Sequences 6 and 7: Post contrast sequences (Fig. A9.1.6)}

20. Leaving the patient unchanged in position in the magnet, inject the contrast agent, flush the line with $10 \mathrm{ml}$ saline, and then immediately run "post contrast" sagittal

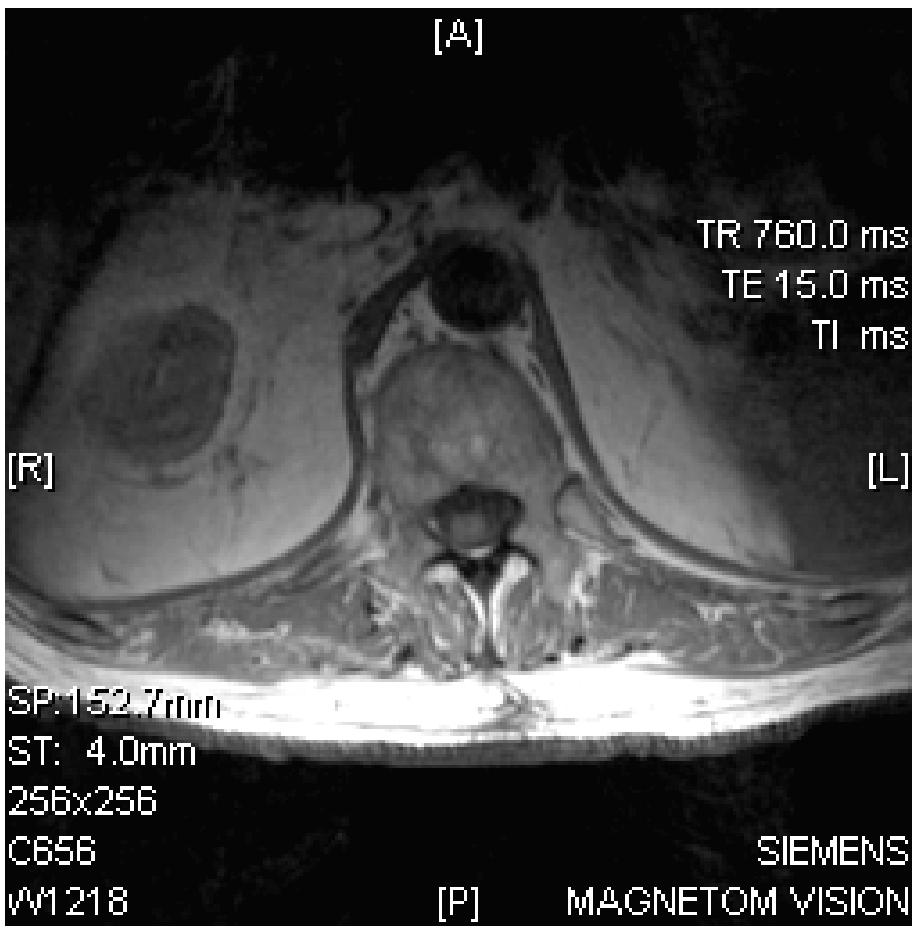

Figure A9.1.5 Transverse $T_{1}$-weighted spin echo image.

Table A9.1.6 Primary Clinical Imaging Parameters for $T_{1}$-Weighted Spin Echo (Sequence 5)

Patient position

Scan type

Imaging plane (orientation)

Central slice or volume center

Echo time $\left(T_{\mathrm{E}}\right)$

Repeat time $\left(T_{\mathrm{R}}\right)$

Flip angle (FA)

Fields of view $\left(\mathrm{FOV}_{\mathrm{x}}, \mathrm{FOV}_{\mathrm{y}}\right)$

Resolution $(\Delta x, \Delta y)$

Number of data points collected $\left(N_{\mathrm{x}}, N_{\mathrm{y}}\right)$

Display matrix $\left(D_{\mathrm{x}}, D_{\mathrm{y}}\right)$

Slice thickness $(\Delta z)$

Number of slices

Slice gap

Number of acquisitions $\left(N_{\text {acq }}\right)$

Flow compensation

Saturation pulses

Scan time
Supine

Conventional spin echo

Transverse

Centered on the area of interest

(as in sequence 2, Table A9.1.3)

$16 \mathrm{msec}$

$500 \mathrm{msec}$

$90^{\circ}$

$200 \mathrm{~mm}, 200 \mathrm{~mm}$

$0.78 \mathrm{~mm}, 1.04 \mathrm{~mm}$

256, 192

256,256

$4 \mathrm{~mm}$

23

$1 \mathrm{~mm}$

3

Yes (if available)

Superior, inferior

$5 \mathrm{~min}, 25 \mathrm{sec}$ 
A

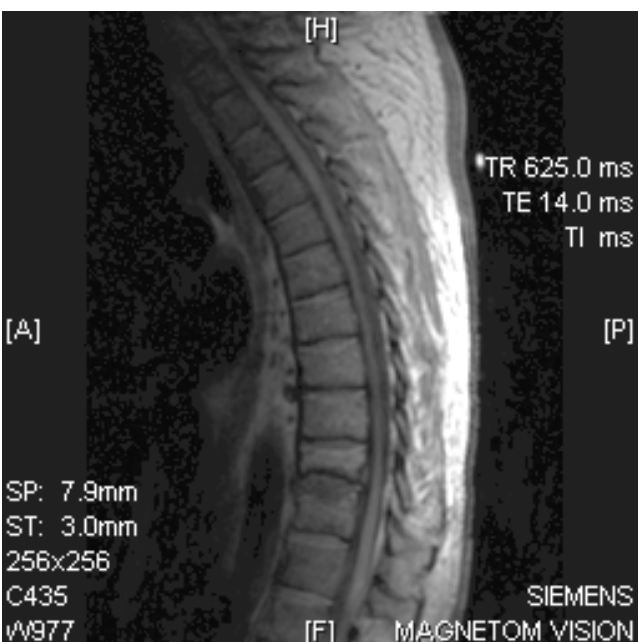

B

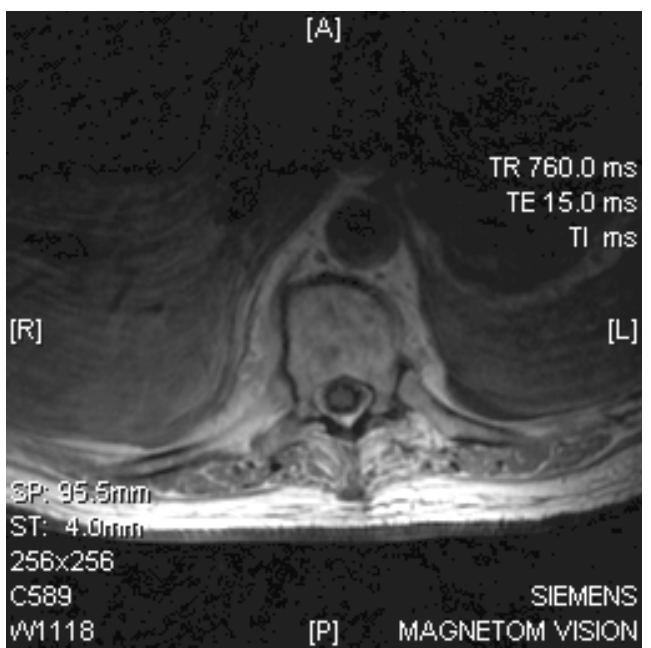

Figure A9.1.6 Post-contrast $T_{1}$-weighted spin echo images: (A) sagittal and (B) transverse.

(sequence 6) and transverse (sequence 7) $T_{1}$-weighted image sequences (using the parameters in Tables A9.1.3 and A9.1.6, respectively).

\section{TRAUMA}

For trauma cases, the most important reason for the examination is to determine if there has been cord hemorrhage. This is best detected using $T_{2}{ }^{*}$-weighted gradient echo images. For the trauma protocol, a sagittal $T_{2}{ }^{*}$-weighted sequence is added (Table A9.1.7) and the enhanced sequences 6 and 7 are dropped.

Table A9.1.7 Primary Clinical Imaging Parameters for $T_{2}{ }^{*}$-Weighted Gradient Echo (Sequence 8)

\begin{tabular}{ll}
\hline Patient position & Supine \\
Scan type & 2-D gradient echo \\
Imaging plane (orientation) & Sagittal \\
Central slice or volume center & Slice centered on area of interest \\
& (as in sequence 2, Table A9.1.3) \\
Echo time $\left(T_{\mathrm{E}}\right)$ & $20 \mathrm{msec}$ \\
Repeat time $\left(T_{\mathrm{R}}\right)$ & $450 \mathrm{msec}$ \\
Flip angle $(\mathrm{FA})$ & $15^{\circ}$ \\
Fields of view $\left(\mathrm{FOV}, \mathrm{FOV}_{\mathrm{y}}\right)$ & $260 \mathrm{~mm}, 260 \mathrm{~mm}$ \\
Resolution $(\Delta x, \Delta y)$ & $1.02 \mathrm{~mm}, 1.35 \mathrm{~mm}$ \\
Number of data points collected $\left(N_{\mathrm{x}}, N_{\mathrm{y}}\right)$ & 256,192 \\
Display matrix $\left(D_{\mathrm{x}}, D_{\mathrm{y}}\right)$ & 256,256 \\
Slice thickness $(\Delta z)$ & $4 \mathrm{~mm}$ \\
Number of slices & 12 \\
Slice gap & $1 \mathrm{~mm}$ \\
Number of acquisitions $\left(N_{\mathrm{acq}}\right)$ & 3 \\
Flow compensation & Yes (if available) \\
Saturation pulses & Superior, inferior, anterior \\
Scan time & $4 \mathrm{~min}, 32$ sec \\
\hline
\end{tabular}


1. Repeat steps 1 to 19 in the Basic Protocol.

\section{Sequence 8: Sagittal $T_{2}{ }^{*}$-weighted gradient echo}

2. Run sequence 8 according to Table A9.1.7.

\section{COMMENTARY}

\section{Background Information}

Just as the gadolinium chelates have proven useful in MR imaging of the brain, the same is true of the spinal cord (Haughton et al., 1999; Najem et al., 1999). Intramedullary tumors and inflammatory processes which are perfused and which have blood-cord barrier breakdown enhance with gadolinium. The most common cord tumor is the glioma of which there are two primary types: astrocytoma and ependymoma (Haughton et al., 1999; Najem et al., 1999). Astrocytomas tend to occur higher in the cord than ependymomas, often involving the cervical region or even extensive portions of the cervical and thoracic cord. They are infiltrative lesions which are difficult to resect surgically and respond poorly to radiation therapy. Ependymomas tend to occur lower in the cord, near the conus medullaris, and are generally better behaved than astrocytomas. Specifically, they are more easily removed surgically and tend to have a more favorable response to radiotherapy. As with other processes in the cord, it is important to define the upper and lower extent of disease. In the case of ependymomas, it is particularly important to image the entire neuraxis as the tumor can seed through the cerebrospinal fluid (CSF) spaces throughout the spinal and intracranial subarachnoid spaces (Haughton et al., 1999; Najem et al., 1999).

Acute tumefactive multiple sclerosis (MS) can simulate a cord tumor. Often, the key to the diagnosis is not the additional administration of gadolinium to the cord lesion but rather an additional MRI of the brain to search for periventricular lesions. We have found thin slice, sagittal, fast FLAIR (fluid attenuated inversion recovery) to be particularly useful for this assessment (Hashemi et al., 1995; Palmer et al., 1999). In the absence of intracranial findings, tumefactive MS may not be distinguishable from cord tumor, either on the basis of enhancement features or lack of same. In such cases, it is useful to rescan the patient in 6 or 12 weeks, as acute tumefactive MS tends to resolve over this period of time while a tumor would tend to remain unchanged in size or to grow.
While MS is, by definition, a relapsing disease, it may be difficult to make the diagnosis definitively on the basis of the initial presentation. Similarly, it may be difficult to distinguish the initial presentation of MS from another demyelinating process such as ADEM (acute disseminated encephalomyelitis). ADEM is a monophasic demyelinating process which is typically found in children and young adults following an exanthematous viral infection or vaccination. It is an autoimmune reaction to the patient's own white matter and can involve either the brain or the spinal cord. It tends to produce somewhat larger lesions than those seen with multiple sclerosis. Since it is immune-mediated, ADEM responds to steroids.

Syringohydromyelia is a difficult diagnosis to make by myelography as the overall cord contour may not be enlarged (Haughton et al., 1999; Najem et al., 1999). Delayed post-myelogram CT (computed tomography) has been useful in demonstrating diffusion of contrast material into the central cavity; however, it is much less sensitive than MRI. When a syrinx is identified on an MR study, its upper and lower margins should be identified. The foramen magnum should be evaluated for the presence of low-positioned cerebellar tonsils, as a Chiari I malformation is often associated with a syrinx. Actually, since this form of syrinx is really enlargement of the ependyma-lined central canal, the more proper term is "hydromyelia". If a syrinx is found without a Chiari I malformation, then gadolinium should be given, as syringes are often found in association with cord tumors. These tumors can be cranial or caudad to the syrinx and can be intra- or extramedullary (Haughton et al., 1999; Najem et al., 1999). They are thought to arise from the obstruction of flow of CSF between the central canal and the subarachnoid space via the perivascular spaces of the cord (Fischbein et al., 1999).

Hemangioblastomas are highly vascular tumors which can not only produce a syrinx but also form a tumor cyst within the cord. When a hemangioblastoma is found within the cord, the cerebellum should also be evaluated as asymptomatic lesions may be harbored there as 
well. When found in both locations, the patient may have von Hippel-Lindau disease.

Trauma can injure the spinal cord through compression from posterior buckling of the posterior longitudinal ligament or frank retropulsion of a fractured vertebral body into the canal (Davis et al., 1991). The key MR finding in spinal trauma is cord hemorrhage. Since hemorrhage may either be identified on the basis of a short $T_{1}$ or a short $T_{2}$, both $T_{1}$-weighted spin echo and $T_{2}{ }^{*}$-weighted gradient echo images should be performed. $\left(T_{2^{-}}\right.$ weighted spin echo images tend to be prone to motion artifact and the commonly used $T_{2^{-}}$ weighted fast spin echo sequences tend to minimize susceptibility effects and the detection of short $T_{2}$ hemorrhage.) Cord edema without hemorrhage (i.e., a "bland contusion") tends to resolve with minimal (if any) neurologic deficits, while hemorrhagic contusions tend to be associated with more serious disability. In the acute or subacute setting, it is also important to exclude persistent causes of cord compression (i.e., extruded fragments or retropulsed bony fragments), which may have been overlooked on plain films or CT. The late sequellae of trauma include myelomalacia (gliosis of the cord), cystic myelomalacia, and frank syrinx formation. In the extreme, cord transection can be easily diagnosed by MR.

MR is useful for diagnosing vascular malformations of the cord of which there are three types: intramedullary arteriovenous malformations (AVMs), extramedullary (radiculomeningeal or dural) AVMs, and cavernous angiomas (Haughton et al., 1999; Najem et al., 1999). As in the brain, cavernous angiomas generally appear as areas of low signal on $T_{2}$-weighted images (due to hemosiderin). In institutions where the bright CSF, low flip angle, gradient echo technique has been replaced by a $T_{2}$-weighted fast spin echo, these magnetic susceptibility effects may be less obvious (as noted above). True AVMs of the cord (i.e., nidus within cord parenchyma) are unusual. Radiculomedullary vascular malformations of the dura and radicular vessels are more common. These may produce scalloping of the protein cord margins. The flow void produced by these enlarged vessels in the subarachnoid space must be distinguished from the normal CSF flow voids noted particularly posterior to the cord. High cord signal on $T_{2}$-weighted images distal to the AVM may represent ischemia secondary to a vascular steal phenomenon.

\section{Critical Parameters and}

\section{Troubleshooting}

The most common problem with spine imaging is CSF motion artifacts. These can simulate vascular flow voids, giving the appearance of arteriovenous malformations, particularly on bright CSF gradient echo acquisitions (e.g., Table A9.1.5 and Table A9.1.7). In such cases, $T_{2}$-weighted fast spin echo imaging has been shown to have particular utility due to the natural flow compensation inherent in the multiple $180^{\circ}$ pulses. Increasing the bandwidth to lower the echo spacing and decreasing the time available for motion dephasing is another trick to decrease these motion artifacts.

Swapping phase encoding and read directions such that the phase encoding direction is craniocaudad rather than anterior-posterior also serves to minimize CSF motion artifacts overlying the cord which could potentially simulate syringohydromyelia.

Patients with severe back pain should be appropriately medicated with morphine or Demerol (rather than merely given sedation) to be able to lie motionless for their study.

\section{Anticipated Results}

MRI of the cord should demonstrate abnormal signal intensity corresponding to clinical symptoms. These symptoms should correlate with the location of the tracts with the cord.

\section{Literature Cited}

Davis, S.J., Teresi, L.M., Bradley, W.G., Ziemba, M.A., and Blaze, A.E. 1991. Cervical spine hyperextension injuries: MR findings. Radiology 180:245-251.

Fischbein, N.J., Dillon, W.P., Cobbs, C., and Weinstein, P.R. 1999. The "presyrinx" state: A reversible myelopathic condition that may precede syringomyelia. A.J.N.R. 20:7-20.

Hashemi, R.H., Bradley, W.G., Chen, D.-Y., Jordan, J.E., Queralt, J.A., Cheng, A.E., and Henrie, J.N. 1995. Suspected multiple sclerosis: MR imaging with a thin-section fast-FLAIR pulse sequence. Radiology 196:505-510.

Haughton, V.M., Daniels, D.L., Czervionke, L.F., Williams, A.L., and Rand, S.D. 1999. Cervical spine. In Magnetic Resonance Imaging 3rd edition (D.D. Stark and W.G. Bradley, eds.) pp.1833-1850. Mosby, St. Louis.

Najem, E.S., Bazan, C. III, and Jinkins, J.R. 1999. Thoracic Spine. In Magnetic Resonance Imaging 3rd edition (D.D. Stark and W.G. Bradley, eds.) pp. 1851-1882. Mosby, St. Louis.

Palmer, S., Bradley, W.G., Chen, D.-Y., and Patel, S. 1999. Subcallosal striations: An early finding of MS on sagittal, thin slice, fast FLAIR images. Radiology 210:149-153.
Intradural Spine

A9.1.11 
Shellock, F.G. 1996. Pocket Guide to MR Procedures and Metallic Objects. Lippincott-Raven, Philadelphia.

\section{Key References}

Shellock, 1996. See above.

Covers a number of important patient management issues related to MR imaging, including recom- mended safety procedures, a list of metallic implants that have been tested for MR compatibility, and a list of other sources on MR safety.

Contributed by William G. Bradley Long Beach Memorial Medical Center Long Beach, California 